\title{
Minisymposium: Finite Volume Schemes for Degenerate Problems
}

\author{
Mazen Saad
}

\section{Description}

This minisymposium was a response to the growing use of finite volume schemes combined with finite element schemes in degenerate problems and was intended to bring together developers and researchers from academia and industry. Degenerate problem arises from multifluid flow in porous media or in medicine model as the breast cancer or bone healing model.

Saturations of oil, gas and water, or densities of cells are positive quantities, hence it is crucial to propose numerical schemes ensuring the physical properties as the positiveness and conservation. The goal of this minisymposium was to shed light on recent advances, for instance, in nonlinear control volume finite element scheme or nonlinear monotone scheme for degenerate problems with anisotropic and heterogeneous diffusion tensor over general meshes.

The talks included in the minisymposium were the following:

- Cindy Guichard. Université Pierre et Marie Curie, Paris (France). A degenerate coupled parabolic problem arising in the study of the root-nutrient system.

- Moustafa Ibrahim. Ecole Centrale de Nantes (France). Nonlinear CVFE scheme for simulating a breast cancer model.

- Mladen Jurak. University of Zagreb (Croatia). On Double Porosity Model for Two-Phase Flow in Porous Media.

- Mazen Saad. Ecole Centrale Nantes (France). Numerical analysis for partially miscible two-phase two component flows in porous media.

\footnotetext{
M. Saad ( $\square)$

Ecole Centrale de Nantes, Laboratoire de Mathématiques Jean Leray, Nantes, France

e-mail: mazen.saad@ec-nantes.fr
} 